\title{
DEVELOPMENT AND IMPLEMENTATION OF VOLUMETRIC COMPRESSION RELAXATION TESTING OF SKELETAL MUSCLE
}

\author{
Anurag J. Vaidya (1), Benjamin B. Wheatley (2)
}

\author{
(1) Biomedical Engineering \\ Bucknell University \\ Lewisburg, PA, USA
}

\author{
(2) Mechanical Engineering \\ Bucknell University \\ Lewisburg, PA, USA
}

\section{INTRODUCTION}

Computational models of human body - such as the Toyota THUMS model - are frequently used in the automobile safety industry [1]. Such models rely on accurate material properties for body tissues. However, the compressive behavior of skeletal muscle is not fully understood yet, particularly regarding the differences in muscle response to various in vivo loading conditions [2]. It is likely that in vivo muscle experiences a variation between confined and unconfined volumetric boundary conditions, but nearly all previous studies investigating passively compressed tissue have focused on muscle in unconfined compression (UC) or fully confined compression (CC) [2, 3, 4]. One study has investigated muscle under anisotropic semi-confined compression (SC) [5]. However, apparatus used by Bol et al. (2016) does not allow testing the effect of interstitial fluid properties on the mechanics of skeletal muscles. Thus, we have developed novel instrumentation that can help to investigate the effects of volumetric boundary conditions (SC and $\mathrm{CC}$ ) on stress relaxation of skeletal muscles. We also present a viscoelastic model that shows how relaxation behavior differs with boundary conditions.

\section{METHODS}

Custom instrumentation was developed to perform $\mathrm{CC}$ and $\mathrm{SC}$ (Figure 1). In SC, the muscle samples are restricted in e1 and compressed in e 2 but were free to expand in e3. In CC, muscle samples are restricted in e 3 and e1, and compressed in e2. The muscle samples are covered with a clear acrylic sheet so that future imaging studies can be performed. Transverse oriented cuboids (average length $=24.9 \pm 1.1$ $\mathrm{mm}$ for $\mathrm{CC}$ and $26.4 \pm 1.7 \mathrm{~mm}$ for SC, height $=8.89 \mathrm{~mm}$ for $\mathrm{CC}$ and SC) were dissected from three porcine Tibialis Anterior (TA) muscle midbelly after connective tissue and fat were removed. The cuboids were frozen for 48 hours and allowed to thaw for 6 hours before testing in order to reduce the effects of rigor mortis seen immediately after sacrifice. For both $\mathrm{CC}$ and SC, two testing configurations were employed: fast-compression $(\dot{\varepsilon}=15 \% / s)$ and slow-compression $(\dot{\varepsilon}=$ $1 \% / s$ ) to $15 \%$ strain with $\mathrm{n}=3$ for both $\mathrm{SC}$ and CC. All samples relaxed for 300 s after compression. Data was collected by a $50 \mathrm{lbf}(222 \mathrm{~N})$ ADMET static load cell at $100 \mathrm{~Hz}$. Muscle samples were hydrated by physiological buffer solution. First Piola-Kirchhoff stress, peak stress, and nominal strain were determined through post-hoc analysis of initial specimen dimensions.

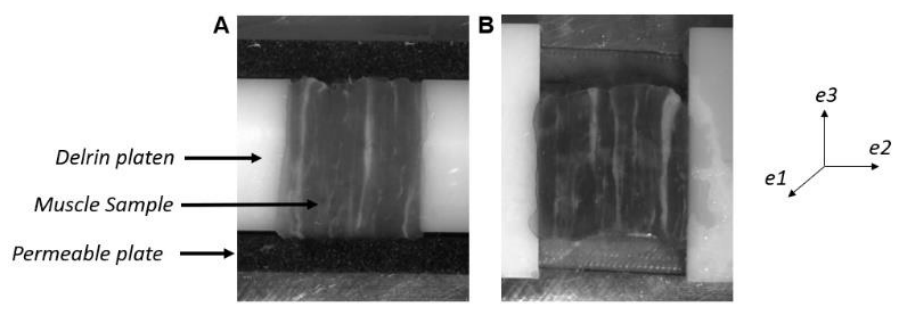

Figure 1: Custom instrumentation developed for (A) fully confined compression (B) Semi-confined compression

To finely characterize relaxation behavior, a three term Prony series quasi-linear viscoelastic model (Equations 1-2) was fit to normalized hold phase stress from all testing groups

$$
\begin{gathered}
P(\varepsilon, t)=\int_{0}^{t} E(t-\tau) \frac{d \varepsilon(\xi)}{d \xi} d \xi \\
E(t)=E_{0}\left(1-\sum_{i=1}^{3} E_{i}\left[1-\exp \left(-\frac{t}{\tau_{i}}\right)\right]\right)
\end{gathered}
$$

Here stress $P$ is calculated from the convolution integral (Equation 2), which includes the Prony series reduced relaxation function $E(t)$, nominal strain $\varepsilon$, and an integration parameter $\xi$. This includes three 
relaxation coefficients $E_{i}$, three time constants $\tau_{i}$, and the instantaneous modulus $E_{0}$. As the purpose of this analysis was to compare relaxation behavior only, $E_{0}=1$ was fixed and all data were normalized. The model also accounted for the experimental overshoot in strain applied by the ADMET during fast compression. Parameter determination was performed in two steps: a Monte Carlo simulation followed by a nonlinear least-squares deterministic optimization (lsqnonlin in MATLAB). In the Monte Carlo simulation, the six parameters $\left(E_{1-3}\right.$ and $\tau_{1-3}$ ) were randomly varied for 100,000 simulations, ensuring $0<$ $E_{1}+E_{2}+E_{3}<1$. The set of parameters minimizing percent error between normalized model and experimental hold stress was used as initial guesses for the deterministic optimization, which optimized percent difference between normalized model and experimental hold stresses. This approach used the global stochastic Monte Carlo method in conjunction with the precision of a local deterministic approach. All modelling was performed in MATLAB (The Mathworks, Inc.). All statistical comparisons between groups were performed using twosample two-tailed t-tests, with significance set to $\mathrm{p}<0.05$. The overall percent error, and normalized root mean square error (NRMSE) were also determined (Equation 3). Here $P_{i}^{\text {mod }}$ and $P_{i}^{\text {exp }}$ are the model and experimental stress values, respectively, at the $\mathrm{i}^{\text {th }}$ data point and $\mathrm{N}$ is the total number of data points.

$$
N R M S E=\frac{\sqrt{\sum_{i=1}^{N}\left(P_{i}^{\text {mod }}-P_{i}^{\text {exp }}\right)^{2}}}{\operatorname{mean}\left(P^{\text {exp }}\right)}
$$

\section{RESULTS}
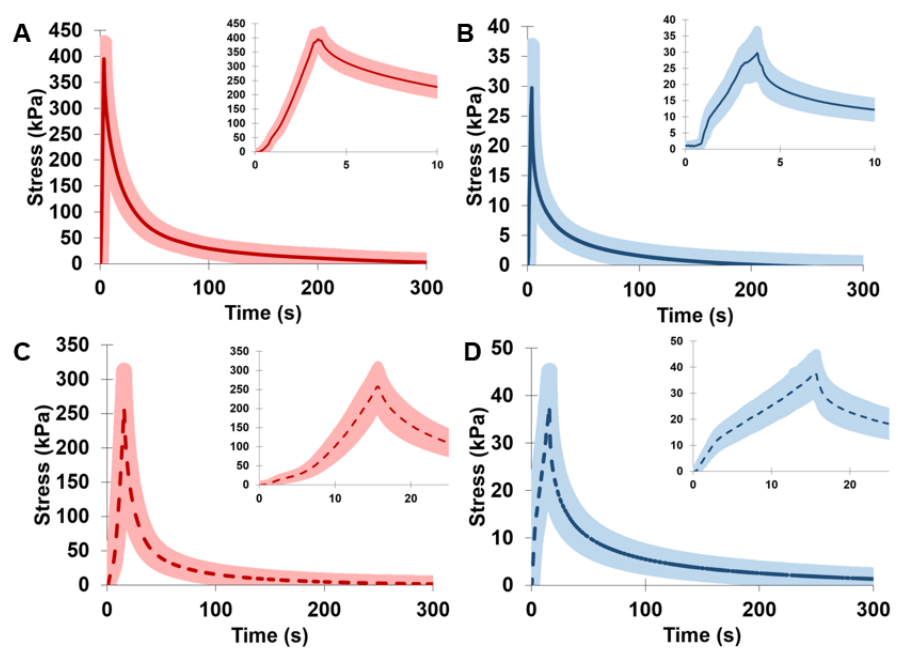

Figure 2: Average stress relaxation curves for (A) CC Fast (B) SC Fast (C) CC Slow (D) SC Slow with standard error bars.

Figure 2 shows that despite similar strain levels, muscle in CC exhibited significantly higher peak stiffness than muscle in SC (p-value<0.001). In $\mathrm{CC}$, muscle stiffness increases with strain rate $(\mathrm{p}$-value $=0.02)$, which is consistent with previous studies [2]. However, muscle's peak stress was not significantly different with strain rate for SC (pvalue $=0.29$ ). Muscle in $\mathrm{CC}$ fast exhibited a more linear ramp behavior than muscle in CC slow. Muscle in SC fast and slow exhibited significant non-linear behavior (Figure 2). The stochastic and deterministic optimization approach provided strong fits between the model and experimental data (Table 1). The parameters from the viscoelastic model (Table 1) go on to support the notion that the relaxation behavior of skeletal muscle is not only rate-dependent, which is consistent with previous studies [3,4], but is also affected by the loading condition.

Table 1: Parameters for the three term Prony series viscoelastic model as applied to the four testing groups along with the overall percent error and NRMSE.

\begin{tabular}{ccccc}
\hline Parameters & CC Fast & CC Slow & SC Fast & SC Slow \\
\hline \multirow{2}{*}{$E_{1-3}$} & $0.37,0.47,0.17$ & $0.35,0.53,0.12$ & $0.46,0.35,0.21$ & $0.67,0.21,0.12$ \\
& & & & \\
$\tau_{1-3}(s)$ & $8.35,25.04$, & $2.75,11.49$, & $0.75,11.94$, & $1.74,15.03$, \\
& 147.4 & 69.81 & 84.3 & 96.58 \\
Percent Error & 1.19 & 3.46 & 6.78 & 1.52 \\
& & & & 0.976 \\
NRMSE & 0.989 & 0.991 & 0.993 \\
\hline
\end{tabular}

\section{DISCUSSION}

This study presents new data of passively compressed muscle under different volumetric boundary conditions (CC versus SC) along with a three term Prony series viscoelastic model. While previous studies have only explored UC or CC, muscle is likely to experience a type of semi-confined loading in vivo. Thus, this study provides data that could be more physiologically accurate and more important gives a broad range of boundary conditions. Moreover, this study compares the $\mathrm{SC}$ data to the extreme loading condition of fully confined compression.

In a previous study done by Vaidya et al. (2020), the peak modulus for muscle in unconfined compression was found to be $\sim 52 \mathrm{kPa}$. While we present a limited sample size, in the current study the peak modulus for $\mathrm{SC}$ fast is $216 \mathrm{kPa}$ and that for CC fast is $2820 \mathrm{kPa}$. The spread of four orders of magnitude present in the peak moduli displays the nonlinear behavior of skeletal muscle under compressive loads. Since $E_{1}$ for SC slow and fast is larger than $E_{1}$ for CC slow and fast and $\tau 1$ is smaller for SC slow and fast, these viscoelastic models suggest that muscle in SC exhibits larger and faster initial relaxation than muscle in $\mathrm{CC}$. Moreover, the fact that $E$ and $\tau$ parameters vary with the strain rate also show that the relaxation behavior of skeletal muscle in CC and SC is rate dependent. The previous study done by Vaidya et al. (2020) showed that muscle in unconfined compression exhibited a $E_{1}$ parameter value of 0.90 and a $\tau 1$ value of $0.132 \mathrm{~s}$. Since the values for $E_{1}$ and $\tau 1$ for SC found in this study fall between those for UC and CC and the peak modulus for SC falls in between that for UC and CC, we conclude that the study successfully presents data on a loading condition (SC) that is somewhere in between fully confined and unconfined compression.

Nevertheless, the present study found that the peak stress of muscle in SC didn't change significantly with the strain rate, which is inconsistent with previous results [3, 4]. This result could have arisen due to a small sample size as well as performing testing after a full cycle of rigor mortis has passed. However, with the current study providing a proof of concept for SC, the authors would like to expand the dataset. The authors would also like to perform digital image correlation on the compression testing as well as test the effect of wetting fluid on the mechanical properties of skeletal muscle in future studies.

\section{REFERENCES}

[1] Toyota Motor Europe. "Toyota THUMS." Toyota Motor Europe

[2] Moh., M., J Mech Behav Biomed Mater 62 (2016): 468-480.

[3] Van Loocke, M., Journal of biomechanics 41.7 (2008): 1555-1566.

[4] Vaidya, A., J Mech Behav Biomed Mater 102 (2020): 103526.

[5] Böl, M., J Mech Behav Biomed Mater 63 (2016): 115-124. 